\title{
Sur les fractions continues des séries formelles quadratiques sur $\mathbb{F}_{q}(X)$
}

\author{
par \\ Mohamed Mkaouar (Sfax)
}

Introduction. Soient $p$ un nombre premier et $\mathbb{F}_{q}$ le corps à $q$ éléments et de caractéristique $p$. L'ensemble $\mathbb{F}_{q}\left(\left(X^{-1}\right)\right)$ représente le corps des séries formelles à coefficients dans $\mathbb{F}_{q}$. Un élément $f \in \mathbb{F}_{q}\left(\left(X^{-1}\right)\right)$ est donc de la forme

$$
f=\sum_{j=s}^{+\infty} f_{j} X^{-j}
$$

où $s \in \mathbb{Z}$. On note par $[f]$ la partie polynomiale $\operatorname{de} f, \operatorname{deg} f=s$ et $\sigma(f)=$ $f_{s}$. Il est clair que si $P$ est un polynôme de degré $n$ dans $\mathbb{F}_{q}[X]$ tel que $P=a_{n} X^{n}+\ldots+a_{0}$, alors $\sigma(P)=a_{n}$. Si $f$ est une série formelle vérifiant l'équation

$$
A_{m} f^{m}+\ldots+A_{1} f+A_{0}=0
$$

où les $A_{k}$ sont des polynômes de $\mathbb{F}_{q}[X]$ non tous nuls, on dit que $f$ est algébrique sur $\mathbb{F}_{q}(X)$. Si de plus l'équation (E) vérifie $\operatorname{deg} A_{k}<\operatorname{deg} A_{m-1}$ pour tout $k \in\{0, \ldots, m\} \backslash\{m-1\}$ et $f$ est de degré $m$, on dit que $f$ est une série formelle algébrique de type (I). Soit $f \in \mathbb{F}_{q}\left(\left(X^{-1}\right)\right)$; alors $f=f_{0}=[f]+(f-[f])$. Soient $a_{0}=\left[f_{0}\right]$ et $f_{1}=(f-[f])^{-1}$, on définit par la suite $f_{n}=a_{n}+f_{n+1}^{-1}$ et $a_{n}=\left[f_{n}\right]$. Finalement, il est clair qu'on peut écrire $f$ sous la forme

$$
f=a_{0}+\frac{1}{a_{1}+\frac{1}{a_{2}+\ldots}}=\left[a_{0} ; a_{1}, a_{2}, \ldots\right] .
$$

Cette nouvelle écriture de $f$ est appelé développement en fraction continue de $f$, et la suite de polynômes $\left(a_{n}\right)_{n \geq 0}$ est appelé suite de quotients partiels de $f$. Il est clair que $\operatorname{deg} a_{i} \geq 1$ pour tout entier strictement positif.

2000 Mathematics Subject Classification: 11A55, 30B70. 
Soit $f$ une série formelle quadratique $\in \mathbb{F}_{q}\left(\left(X^{-1}\right)\right)$. Alors la suite de quotients partiels de son développement en fraction continue est ultimement périodique (i.e. $f=\left[b_{0} ; b_{1}, \ldots, b_{s}, \overline{a_{1}, \ldots, a_{t}}\right]$ ). Nous poserons $T(f)=$ $\max _{1 \leq i \leq t} \operatorname{deg} a_{i}$ et $\operatorname{Per}(f)=t$ sa période. On dit aussi qu'une série formelle quadratique $f$ est purement périodique si sa suite de quotients partiels est périodique (i.e. $f=\left[a_{1} ; \overline{a_{2}, \ldots, a_{t}, a_{1}}\right]$ ); si de plus $f=\left[a_{1} ; \overline{a_{2}, \ldots, a_{t}, a_{1}}\right]$ ) $\left.=\left[a_{t} ; \overline{a_{t-1}, \ldots, a_{1}, a_{t}}\right]\right)$, on dit que $f$ est palindromique.

Dans le cas réel Schinzel [8] a montré l'existence de la quantité

$$
S(N, n)=\sup _{\operatorname{Per}(x)=n} \operatorname{Per}(N x),
$$

où $x$ est un nombre réel quadratique. Un peu plus tard Cohen [4] a redémontré l'existence de $S(N, n)$; de plus, il a évalué la quantité $S(N, n)$, il a prouvé l'existence de

$$
R(N)=\sup _{n \geq 1}\left(\frac{S(N, n)}{n}\right)=\sup _{x \in \mathbb{Q}}\left(\frac{\operatorname{Per}(N x)}{\operatorname{Per}(x)}\right),
$$

et il a donné la valeur exacte de $R(N)$ pour une infinité d'entiers $N$. D'autre part Cohn a étudié dans [5] la longueur de la période du développement en fraction continue de $d^{1 / 2}$, où $d$ est un entier qui n'est pas un carré parfait; il a montré que

$$
\operatorname{Per}(\sqrt{d})<\frac{7}{2 \pi^{2}} \sqrt{d} \log d+O(\sqrt{d}) .
$$

Dans [6] Lewittes a caractérisé les nombres réels quadratiques qui sont palindromiques et il a évalué le nombre $A(n)$ des réels qui sont palindromiques et dont le discriminant est égal à $n$.

Dans le cas des séries formelles, il est démontré dans [7] que si $f$ est une série formelle quadratique sur $\mathbb{F}_{2}(X)$ vérifiant $A f^{2}+B f+C=0$, alors $\operatorname{Per}(f) \leq\left(2^{\operatorname{deg} B}-1\right)^{2}$.

L'objectif de ce travail est de donner dans le cas des séries formelles l'équivalent du théorème de Galois pour le cas quadratique : à savoir la caractérisation des séries formelles quadratiques qui admettent un développement en fraction continue purement periodique. D'autre part, on généralise le résultat établi par Mkaouar [7] sur $\mathbb{F}_{2}\left(\left(X^{-1}\right)\right)$, dans $\mathbb{F}_{q}\left(\left(X^{-1}\right)\right)$, où la caractéristique $p$ du corps $>2$ est de donner un majorant à la période d'une série formelle quadratique de type (I) en fonction des coefficients de son polynôme minimal. De plus, si $p \geq 3$, on donne une condition nécessaire et suffisante pour qu'un polynôme $Q \in \mathbb{F}_{q}[X]$ admet une racine carré dans $\mathbb{F}_{q}\left(\left(X^{-1}\right)\right)$ et que la période du développement en fraction continue de celleci est un $O\left(q^{2 \operatorname{deg} Q}\right)$. Finalement, on donne une caractérisation des séries formelles quadratiques palindromiques. 


\section{Enoncé des résultats}

ThÉORÈme 1. Soit $f$ une série formelle quadratique sur $\mathbb{F}_{q}(X)$ et $[f]$ $\neq 0$. Alors $f$ admet un développement en fraction continue purement périodique si et seulement si $f$ est de type (I).

THÉORÈme 2. Soit $f$ une série formelle quadratique sur $\mathbb{F}_{q}(X)$ de type (I), vérifiant $A f^{2}+B f+C=0$. Alors

$$
\operatorname{Per}(f) \leq\left(q^{\operatorname{deg} B}-1\right)^{2} q^{2 \operatorname{deg} B} \quad \text { et } \quad T(f) \leq \operatorname{deg} B .
$$

ThÉORÈme 3. Soit $f$ une série formelle algébrique de type (I) et de degré $m$ sur $\mathbb{F}_{q}(X)$, telle que $[f] \neq 0$ et $A_{m} f^{m}+\ldots+A_{1} f+A_{0}=0$. Alors $[f]=-\left[A_{m-1} / A_{m}\right]$ et la série formelle $h=1 /(f-[f])$ est une série formelle de type (I).

ThÉORÈme 4 . Soient $q \geq 3$ et $Q$ un polynôme de degré pair avec $\sigma(Q)$ un carré parfait. Alors $Q$ admet au moins une racine carrée $f \in \mathbb{F}_{q}\left(\left(X^{-1}\right)\right)$; de plus si $Q$ n'est pas un carré parfait dans $\mathbb{F}_{q}[X]$, alors toute racine carrée $f$ de $Q$ vérifie

$$
\operatorname{Per}(f) \leq q^{2 \operatorname{deg} Q} \quad \text { et } \quad T(f) \leq \frac{1}{2} \operatorname{deg} Q .
$$

ThÉORÈme 5. Soit $f$ une série formelle quadratique sur $\mathbb{F}_{q}(X)$. Alors $f$ est palindromique si et seulement si elle vérifie une équation de type $A f^{2}+$ $B f-A=0$ avec $A, B \in \mathbb{F}_{q}[X]$ et $\operatorname{deg} B>\operatorname{deg} A$. De plus si $A(n, q)$ désigne le nombre de séries formelles palindromiques dont le discriminant est de degré $2 n$, alors

$A(n, q)=O\left(\frac{q^{2 n}}{n^{\alpha} \sqrt{\log 2 n}}\right) \quad$ avec $\alpha=1-\frac{1+\log \log 2}{\log 2} \quad$ si $p \equiv 1(\bmod 4)$ et

$$
A(n, q)=O\left(\frac{q^{2 n}}{\sqrt{n}}\right) \quad \text { si } p \equiv 3(\bmod 4) \text {. }
$$

Démonstration du théorème 3. Posons $g=f-[f]$. Alors

$$
\begin{aligned}
0 & =\sum_{j=0}^{m} A_{j}([f]+g)^{j} \\
& =\sum_{j=0}^{m} A_{j} \sum_{k=0}^{j}\left(\begin{array}{l}
j \\
k
\end{array}\right)[f]^{j-k} g^{k}=\sum_{k=0}^{m}\left(\sum_{j=k}^{m} A_{j}\left(\begin{array}{l}
j \\
k
\end{array}\right)[f]^{j-k}\right) g^{k} .
\end{aligned}
$$

Soit

$$
C_{k}=\sum_{j=k}^{m} A_{j}\left(\begin{array}{l}
j \\
k
\end{array}\right)[f]^{j-k} .
$$

Comme $\operatorname{deg} f>0$ alors $\operatorname{deg} A_{k} f^{k}<\operatorname{deg} A_{m-1} f^{m-1}$ pour $k<m-1$, et par suite d'après (E), $\operatorname{deg} A_{m} f^{m}=\operatorname{deg} A_{m-1} f^{m-1}$. Ceci donne $\operatorname{deg} f=$ 
$\operatorname{deg} A_{m-1}-\operatorname{deg} A_{m}$ et

$$
f=-A_{m-1} / A_{m}-\sum_{j=1}^{m-1} l_{j}
$$

avec $l_{j}=A_{m-j-1} / A_{m} f^{j}$. D'où

$$
\operatorname{deg} l_{j}=\operatorname{deg} A_{m-j-1}+(j-1) \operatorname{deg} A_{m}-j \operatorname{deg} A_{m-1}<0,
$$

ce qui donne que $[f]=-\left[A_{m-1} / A_{m}\right]$.

Soit $h=1 /(f-[f])$, alors la série formelle $h$ vérifie l'équation

$$
\sum_{k=0}^{m} C_{m-k} h^{k}=0
$$

Comme $f$ est algébrique et degré $m$, alors $h$ l'est aussi. Donc pour montrer que $h$ est de type (I), il suffit de montrer que $\operatorname{deg} C_{1}>\operatorname{deg} C_{k}$ pour tout $k \in\{0, \ldots, m\} \backslash\{1\}$. Comme $[f]=-\left[A_{m-1} / A_{m}\right]$, alors $A_{m-1}=-[f] A_{m}+R$ avec $\operatorname{deg} R<\operatorname{deg} A_{m}$. D'après (1),

$$
C_{0}=\sum_{j=0}^{m} A_{j}[f]^{j}=\left(\sum_{j=0}^{m-2} A_{j}[f]^{j}\right)+R[f]^{m-1}
$$

et

$$
C_{1}=\sum_{j=0}^{m} j A_{j}[f]^{j-1}=\left(\sum_{j=0}^{m-2} j A_{j}[f]^{j-1}\right)+A_{m}[f]^{m-1}+(m-1) R[f]^{m-2} .
$$

Il est clair que $\operatorname{deg} C_{1}=(m-2) \operatorname{deg}[f]+\operatorname{deg} A_{m-1}$ et que $\operatorname{deg} C_{0} \leq$ $(m-2) \operatorname{deg}[f]+\operatorname{deg} A_{m-1}$. Comme $\operatorname{deg} C_{k} \leq \operatorname{deg} A_{m-1}+(m-k-1) \operatorname{deg}[f]$ pour tout $k \in\{1, \ldots, m\}$, alors, pour tout $k \in\{1, \ldots, m\} \backslash\{1\}$, on a $\operatorname{deg} C_{k}<\operatorname{deg} C_{1}$.

\section{Application}

Corollaire 1. Soient $n \in \mathbb{N}^{*}$ et $f$ une série formelle algébrique sur $\mathbb{F}_{q}(X)$ vérifiant $[f] \neq 0$ et $A f^{q^{n}+1}+A B f^{q^{n}}+1=0$, avec $\operatorname{deg} B>0$. Alors $f=\left[a_{0} ; a_{1}, \ldots\right]$, ò̀

$$
a_{s}=(-1)^{s} A^{\left(q^{s n}-(-1)^{s}\right) /\left(q^{s}+1\right)} B^{q^{s n}} .
$$

Preuve. Soit $f_{0}=f, a_{0}=\left[f_{0}\right], P_{0}=A, Q_{0}=A B, R_{0}=0, S_{0}=1$ et $f_{s+1}=1 /\left(f_{s}-\left[f_{s}\right]\right)$. Alors d'après le théorème $3, f_{s}$ est de type (I) et vérifie l'équation $P_{s} f_{s}^{q^{n}+1}+Q_{s} f_{s}^{q^{n}}+R_{s} f_{s}+S_{s}=0$, avec

$$
\begin{aligned}
& P_{s+1}=P_{s} a_{s}^{q^{n}+1}+Q_{s} a_{s}^{q^{n}}+R_{s} a_{s}+S_{s}, \quad Q_{s+1}=P_{s} a_{s}^{q^{n}}, \\
& R_{s+1}=Q_{s}+a_{s} P_{s}, \quad S_{s+1}=P_{s}, \quad a_{s+1}=-\left[\frac{Q_{s+1}}{P_{s+1}}\right] .
\end{aligned}
$$


A l'aide d'une récurrence simple sur $s$ on montre que

$$
\begin{aligned}
P_{s}=A^{\left(1+(-1)^{s}\right) / 2}, & Q_{s}=(-1)^{s} A^{\left(1-(-1)^{s}\right) / 2} A^{\left(q^{s n}+(-1)^{s} q^{n}\right) /\left(q^{n}+1\right)} B^{q^{s n}} \\
& R_{s}=0, \quad S_{s}=A^{\left(1-(-1)^{s}\right) / 2}
\end{aligned}
$$

et que

$$
a_{s}=(-1)^{s} A^{\left(q^{s n}+(-1)^{s}\right) /\left(q^{n}+1\right)} B^{q^{s n}} .
$$

Corollaire 2 ([1], théorème 6$)$. Soit $n \in \mathbb{N}$. Alors l'équation

$$
f^{2^{n}+1}+Q f^{2^{n}}+P f+P Q+1=0
$$

admet une racine unique dans $\mathbb{F}_{2}\left(\left(X^{-1}\right)\right)$, et

$$
f=\left[Q ; Q^{2^{2 n}}+P^{2^{n}}, Q^{2^{3 n}}+P^{2^{2 n}}, \ldots\right] .
$$

Preuve. Il suffit de remarquer que la série formelle $g=1 /(f-Q)$ vérifie

$$
g^{2^{n}+1}+\left(Q^{2^{n}}+P\right) g^{2^{n}}+1=0
$$

d'après le corollaire 1 : il suffit de prendre $A=1, B=Q^{2^{n}}+P$ et $q=2$.

Démonstration du théorème 1 . Ici, on donne l'équivalent du théorème de Galois pour le cas quadratique sur $\mathbb{F}_{q}\left(\left(X^{-1}\right)\right)$. Il est clair que si $f$ est périodique alors $f$ vérifie l'équation $Q_{n} f^{2}+\left(Q_{n-1}-P_{n}\right) f-P_{n-1}=0$, où $f=\left[a_{1} ; \overline{a_{2}, \ldots, a_{t}, a_{1}}\right]$ et $P_{n} / Q_{n}=\left[a_{1} ; a_{2}, \ldots, a_{n}\right]$. On a alors $\operatorname{deg} Q_{n}<$ $\operatorname{deg}\left(P_{n}-Q_{n-1}\right)=\operatorname{deg} P_{n}$ et $\operatorname{deg} P_{n-1}<\operatorname{deg} P_{n}$.

On suppose maintenant que $f$ est de type (I) et vérifie $A f^{2}+B f$ $+C=0$. Alors $f$ est ultimement périodique, donc $f$ est de la forme $f=$ $\left[a_{1} ; a_{2}, \ldots, a_{t}, f_{t+1}\right]$, où $f_{t+1}$ est une série formelle périodique. Soit $f=f_{1}$ et $f_{n+1}=1 /\left(f_{n}-a_{n}\right)$. Comme $f$ est de type (I), alors d'après le théorème 3 , $f_{n}$ l'est aussi et vérifie l'équation $H_{n} f_{n}^{2}+K_{n} f_{n}+H_{n-1}=0$, avec $H_{0}=C$, $H_{1}=A, K_{1}=B, a_{1}=-\left[K_{1} / H_{1}\right]$, et

$$
\begin{gathered}
H_{n+1}=a_{n}^{2} H_{n}+a_{n} K_{n}+H_{n-1}, \\
K_{n+1}=2 a_{n} H_{n}+K_{n}, \\
a_{n+1}=-\left[K_{n+1} / H_{n+1}\right] .
\end{gathered}
$$

Soit $\operatorname{Per}(f)=\operatorname{Per}\left(f_{t+1}\right)=l$. Alors $H_{t+j}=H_{t+l+j}, K_{t+j}=K_{t+l+j}$ et $a_{t+j}=a_{t+j+l}$, pour tout $j \geq 1$. Ceci donne d'après la formule de récurrence (2) pour $H_{t+l+2}$ et $H_{t+2}$ que

$$
H_{t}=H_{t+l}
$$

Par suite,

$$
K_{t+l+1}=2 a_{t+l} H_{t+l}+K_{t+l}=2 a_{t+l} H_{t}+K_{t+l}=K_{t+1}=2 a_{t} H_{t}+K_{t},
$$


ce qui donne

$$
2\left(a_{t+l}-a_{t}\right) H_{t}=K_{t}-K_{t+l}
$$

Soit alors

$$
K_{t}=\left[K_{t} / H_{t}\right] H_{t}+R_{t}=-a_{t} H_{t}+R_{t},
$$

avec $\operatorname{deg} R_{t}<\operatorname{deg} H_{t}$, et

$$
K_{t+l}=\left[K_{t+l} / H_{t+l}\right] H_{t+l}+R_{t+l}=-a_{t+l} H_{t+l}+R_{t+l},
$$

avec $\operatorname{deg} R_{t+l}<\operatorname{deg} H_{t}$. En remplaçant $K_{t+l}$ et $K_{t}$ par leurs expressions dans (6), on obtient

$$
\left(a_{t+l}-a_{t}\right) H_{t}=R_{t}-R_{t+l} .
$$

Comme $\operatorname{deg}\left(R_{t}-R_{t+l}\right)<\operatorname{deg} H_{t}$, (9) nous permet de dire que $a_{t}=a_{t+l}$ et $R_{t}=R_{t+l}$, ce qui donne d'après (5), (7) et (8), $K_{t}=K_{t+l}$ et par suite d'après les formules de récurrence (2), (3) et (4), $H_{t-1}=H_{t-1+l}, \ldots, H_{1}=$ $H_{1+l}, K_{t-1}=K_{t-1+l}, \ldots, K_{1}=K_{1+l}$ et $a_{t-1}=a_{t-1+l}, \ldots, a_{1}=a_{1+l}$, ce qui montre que $f$ est périodique.

Démonstration du théorème 2. Pour la démonstration de ce théorème, on a besoin du lemme suivant :

LEMME 1. Soit $f$ une série formelle de type (I) vérifiant $(\mathrm{E}): A f^{2}+$ $B f+C=0$. Alors $f$ et $-(f+B / A)$ sont les seules solutions de $(\mathrm{E})$. De plus si $f=\left[a_{1} ; \overline{a_{2}, \ldots, a_{n}, a_{1}}\right]$, alors $-(f+B / A)=\left[0 ; \overline{-a_{n},-a_{n-1}, \ldots,-a_{1}}\right]$.

Preuve. Il est facile de vérifier que si $f$ est solution de (E), alors $-(f+B / A)$ l'est aussi, donc on peut supposer sans perte de généralité que $[f] \neq 0$. Dans ce cas d'après le théorème $3,[f]=-[B / A]=a_{1}$. Soit $P_{n} / Q_{n}=\left[a_{1} ; a_{2}, \ldots, a_{n}\right]$. Il est clair que

$$
f=\left[a_{1} ; a_{2}, \ldots, a_{n}, f\right]=\frac{P_{n} f+P_{n-1}}{Q_{n} f+Q_{n-1}},
$$

ce qui donne $Q_{n} f^{2}+\left(Q_{n-1}-P_{n}\right) f-P_{n-1}=0$. On a $P_{n-1} / P_{n}=\left[0 ; a_{n}, a_{n-1}\right.$, $\left.\ldots, a_{1}\right]$ et $Q_{n-1} / Q_{n}=\left[0 ; a_{n}, \ldots, a_{2}\right]$. Posons $h=\left[0 ; \overline{-a_{n}, \ldots,-a_{1}}\right]$; alors $h=\left[0 ;-a_{n}, \ldots,-a_{1}+h\right]$, ce qui donne $-h=\left(P_{n-1}-Q_{n-1} h\right) /\left(P_{n}-Q_{n} h\right)$ et par suite $h$ vérifie l'équation $Q_{n} h^{2}+\left(Q_{n-1}-P_{n}\right) h-P_{n-1}=0$. Comme $f$ vérifie la même équation que $h$, elles sont conjuguées et par conséquent $h=-(f+B / A)$.

Suite de la démonstration du théorème 2. Soit $f \in \mathbb{F}_{q}\left(\left(X^{-1}\right)\right)$ de type (I) vérifiant $A f^{2}+B f+C=0$. On peut supposer que $[f] \neq 0$. Soit $f=\left[a_{1} ; \overline{a_{2}, \ldots, a_{l}, a_{1}}\right], f=f_{1}$ et $f_{n+1}=1 /\left(f_{n}-a_{n}\right)$. Alors d'après le théorème 3 , pour tout $n \in \mathbb{N}^{*}$, la série $f_{n}$ est de type (I) et elle vérifie l'équation

$$
H_{n} f_{n}^{2}+K_{n} f_{n}+H_{n-1}=0
$$


où les suites de polynômes $H_{n}$ et $K_{n}$ sont définies par les relations de récurrences données par (2), (3) et (4). La division euclidienne de $K_{n}$ par $H_{n}$ donne $K_{n}=-a_{n} H_{n}+R_{n}$ avec $\operatorname{deg} R_{n}<\operatorname{deg} H_{n}$. Or (3) nous permet de dire que $K_{n+1}=-K_{n}+2 R_{n}$, ce qui donne que pour tout entier positif $n$,

$$
\operatorname{deg} K_{n}=\operatorname{deg} B \text {. }
$$

Comme $f$ est de type (I), alors d'après le théorème $1, f$ est purement périodique, donc d'après (10), la suite $W_{n}=\left(H_{n}, K_{n}\right)$ l'est aussi et partage la même période $l$ que $f$. Soit $t \in \mathbb{N}$; alors $W_{t+l}=W_{t}$ et $W_{t+l+1}=W_{t+1}$. Soit donc $s \in \mathbb{N}^{*}$ le plus petit entier tel que $W_{t+s}=W_{t}$ et $W_{t+s+1}=W_{t+1}$. Les formules de récurrences qui relient $H_{n+1}$ et $K_{n+1}$ à $H_{n}, K_{n}, a_{n}$ montrent que $H_{t+s+2}=H_{t+2}, H_{t+s+3}=H_{t+3}, \ldots, H_{t+2 s}=H_{t+s}, K_{t+s+2}=K_{t+2}$, $K_{t+s+3}=K_{t+3}, \ldots$, et que $K_{t+2 s}=K_{t+s}$. Ceci montre que $s$ est la période de la suite $W_{n}=\left(H_{n}, K_{n}\right)$ et par suite $s=l$. Comme $H_{n} \neq 0$ et d'après (10), $\operatorname{deg} H_{n}<\operatorname{deg} K_{n}=\operatorname{deg} B$, le nombre de termes distincts de la suite $W_{n}$ est majoré par $\left(q^{\operatorname{deg} B}-1\right) q^{\operatorname{deg} B}$. Alors le nombre de couples $\left(W_{n}, W_{n+1}\right)$ distincts est majoré par $\left(q^{\operatorname{deg} B}-1\right)^{2} q^{2 \operatorname{deg} B}$. Donc dans les $\left(q^{\operatorname{deg} B}-1\right)^{2} q^{2 \operatorname{deg} B}+1$ premiers termes de la suite $\left(W_{n}, W_{n+1}\right)$, on trouve deux couples identiques $\left(W_{t}, W_{t+1}\right)=\left(W_{t+s}, W_{t+s+1}\right)$, ce qui donne $l=s \leq\left(q^{\operatorname{deg} B}-1\right)^{2} q^{2 \operatorname{deg} B}$. D'autre part, pour tout entier strictement positif $n$ on a $\operatorname{deg} a_{n}=\operatorname{deg} K_{n}-\operatorname{deg} H_{n}$, et d'après (10), on déduit que $T(f) \leq \operatorname{deg} B$.

Démonstration du théorème 4. Pour la démonstration de ce théorème, on a besoin de quelques lemmes.

Lemme 2. Soit $q \geq 2$. Alors l'équation (E) : $A Y^{2}+B Y+C=0$ avec $A, B, C \in \mathbb{F}_{q}[X] \backslash\{0\}$ et $\operatorname{deg} B>\operatorname{deg} A, \operatorname{deg} C$ admet au moins une racine $f$ dans $\mathbb{F}_{q}\left(\left(X^{-1}\right)\right)$ telle que $\operatorname{deg}[f] \geq 1$.

Preuve. Soient $H_{n}, K_{n}$ et $a_{n}$ les suites de polynômes définies par $H_{0}=$ $C, H_{1}=A, K_{1}=B$ et $a_{1}=-\left[K_{1} / H_{1}\right], H_{n+1}=a_{n}^{2} H_{n}+a_{n} K_{n}+H_{n-1}$, $K_{n+1}=2 a_{n} H_{n}+K_{n}$ et $a_{n+1}=-\left[K_{n+1} / H_{n+1}\right]$. Il est clair que les termes $H_{n+1}, K_{n+1}$ et $a_{n+1}$ ne sont définis que si $H_{n} \neq 0$. Pour cela, on suppose que $H_{n} \neq 0$ pour $n \leq m$. Soient $P_{0}=1, Q_{0}=0, P_{n} / Q_{n}=\left[a_{1}, \ldots, a_{n}\right]$ et

$$
\begin{aligned}
& U_{n}=A P_{n}^{2}+B P_{n} Q_{n}+C Q_{n}^{2}, \\
& V_{n}=2 P_{n} P_{n-1} A+\left(P_{n} Q_{n-1}+Q_{n} P_{n-1}\right) B+2 Q_{n} Q_{n-1} C .
\end{aligned}
$$

Montrons que pour tout $n \in\{1, \ldots, m\}$,
$\left(\alpha_{n}\right)$
$U_{n}=H_{n+1}$,$$
V_{n}=K_{n+1}
$$

Les propriétés $\left(\alpha_{n}\right)$ et $\left(\beta_{n}\right)$ sont vraies pour $n=1$. On les suppose vraies pour $n<s \leq m$. Alors en utilisant le fait que pour tout $s \in \mathbb{N}, P_{s}=$ 


$$
\begin{aligned}
a_{s} P_{s-1} & +P_{s-2} \text { et } Q_{s}=a_{s} Q_{s-1}+Q_{s-2}, \text { on aura } \\
U_{s}= & A\left(a_{s} P_{s-1}+P_{s-2}\right)^{2}+B\left(a_{s} P_{s-1}+P_{s-2}\right)\left(a_{s} Q_{s-1}+Q_{s-2}\right) \\
& +C\left(a_{s} Q_{s-1}+Q_{s-2}\right)^{2} \\
= & a_{s}^{2}\left(A P_{s-1}^{2}+B P_{s-1} Q_{s-1}+C Q_{s-1}^{2}\right)+\left(A P_{s-2}^{2}+B P_{s-2} Q_{s-2}+C Q_{s-2}^{2}\right) \\
& \quad+a_{s}\left(2 A P_{s-1} P_{s-2}+B\left(P_{s-1} Q_{s-2}+Q_{s-1} P_{s-2}\right)+2 C Q_{s-1} Q_{s}\right) \\
= & a_{s}^{2} H_{s}+a_{s} K_{s}+H_{s-1} \\
= & H_{s+1} .
\end{aligned}
$$

De même

$$
\begin{aligned}
V_{s}= & 2\left(a_{s} P_{s-1}+P_{s-2}\right) P_{s-1} A+2\left(a_{s} Q_{s-1}+Q_{s-2}\right) Q_{s-1} C \\
& +\left(\left(a_{s} P_{s-1}+P_{s-2}\right) Q_{s-1}+\left(a_{s} Q_{s-1}+Q_{s-2}\right) P_{s-1}\right) B \\
= & 2 a_{s}\left(P_{s-1}^{2} A+P_{s-1} Q_{s-1} B+Q_{s-1}^{2} C\right)+2 Q_{s-2} Q_{s-1} C \\
& +2 P_{s-2} P_{s-1} A+\left(P_{s-2} Q_{s-1}+Q_{s-2} P_{s-1}\right) B \\
= & 2 a_{s} H_{s}+K_{s} \\
= & K_{s+1} .
\end{aligned}
$$

Il est clair maintenant que si $A_{n} \neq 0$ pour tout $n \leq m$, alors

$$
A\left(\frac{P_{m}}{Q_{m}}\right)^{2}+B\left(\frac{P_{m}}{Q_{m}}\right)+C=\frac{1}{Q_{m}^{2}} A_{m+1} .
$$

- S'il existe $m \geq 1$ tel que $A_{n} \neq 0$ pour tout $n \leq m$ et $A_{m+1}=0$, alors

$$
A\left(\frac{P_{m}}{Q_{m}}\right)^{2}+B\left(\frac{P_{m}}{Q_{m}}\right)+C=0,
$$

ce qui donne que $\left(P_{m} / Q_{m}\right)=\left[a_{1} ; \ldots, a_{m}\right]$ est solution de $(\mathrm{E})$.

- Si $A_{n} \neq 0$ pour tout $n \in \mathbb{N}$, alors on considère la série formelle $f=$ $\lim \left(P_{n} / Q_{n}\right)=\lim \left[a_{1} ; \ldots, a_{n}\right]$, et par conséquent

$$
\begin{aligned}
A f^{2}+B f+C & =\lim A\left(\frac{P_{n}}{Q_{n}}\right)^{2}+B\left(\frac{P_{n}}{Q_{n}}\right)+C \\
& =\lim \frac{1}{Q_{n}^{2}} A_{n+1} \\
& =0 \quad\left(\text { car pour tout } n \in \mathbb{N}, \operatorname{deg} A_{n}<\operatorname{deg} B\right) .
\end{aligned}
$$

Lemme 3. Soient $q \geq 3$ et $Q$ un polynôme dans $\mathbb{F}_{q}[X]$. Alors les deux assertions suivantes sont équivalentes :

(i) $\operatorname{deg} Q$ est pair et $\sigma(Q)$ est un carré parfait.

(ii) Il existe deux couples distincts $(T, S) \in \mathbb{F}_{q}[X]^{2}$ tels que $\operatorname{deg} T>\operatorname{deg} S$ et $Q=T^{2}-S$. 
Preuve. (i) $\Rightarrow($ ii). Soit $\varrho$ une racine carré de $\sigma(Q)$. Posons

$$
\begin{aligned}
& Q=\sum_{k=0}^{2 n} \alpha_{k} X^{k}, \quad \alpha_{2 n}=\varrho^{2}, \\
& T_{\varrho}=\sum_{k=0}^{n} \beta_{k} X^{k}, \quad \beta_{n}=\varrho .
\end{aligned}
$$

Dire que $\operatorname{deg}\left(Q-T_{\varrho}^{2}\right)<\operatorname{deg} T_{\varrho}$ signifie pour tout $k \in\{n, n+1, \ldots, 2 n\}$,

$$
\sum_{\substack{0 \leq i \leq j \leq n \\ i+j=k}}\left(2-\varepsilon_{i j}\right) \beta_{i} \beta_{j}=\alpha_{k},
$$

où $\varepsilon_{i j}$ vaut 1 si $i=j$ et 0 sinon. Si on suppose les $\beta_{t}$ connus pour $s \leq t \leq n$, alors on sait déterminer d'une manière unique $\beta_{s-1}$ si $s>0$. En effet, d'après $(11)$

$$
2 \beta_{s-1}=\alpha_{n+s-1}-\sum_{\substack{0 \leq i \leq j<n \\ i+j=n+s-1}}\left(2-\varepsilon_{i j}\right) \beta_{i} \beta_{j},
$$

or d'après notre supposition, tous les $\beta_{i}, \beta_{j}$ sont connus dès que $0 \leq i \leq j$ $<n$ et $i+j=n+s-1$, ce qui détermine entièrement les coefficients de $T_{\varrho}$ en fonction de ceux de $Q$. L'existence de deux couples $(T, S)$ vérifiant (ii) vient du fait que si $\sigma(Q)$ est un carré parfait $\in \mathbb{F}_{q}$, alors les seules racines carrées de $\sigma(Q)$ sont $\varrho$ et $q-\varrho$ et par conséquent $\left(T_{\varrho}, S_{\varrho}\right)$ et $\left(T_{q-\varrho}, S_{q-\varrho}\right)$ sont les seuls couples vérifiant (ii).

(ii) $\Rightarrow\left(\right.$ i). Il est clair que $\operatorname{deg} Q=2 \operatorname{deg} T$ et que $\sigma(Q)=\sigma\left(T^{2}\right)=\sigma^{2}(T)$.

Suite de la démonstration du théorème 4. Soit $Q$ un polynôme de degré pair et $\sigma(Q)$ est un carré parfait. Alors d'après le lemme 3, il existe $T, S \in$ $\mathbb{F}_{q}[X]$ tels que $\operatorname{deg} T>\operatorname{deg} S$ et $Q=T^{2}-S$. D'après le lemme 2 , l'équation $(\mathrm{F}): S Y^{2}+2 T Y+1=0$ admet au moins une racine $g$ dans $\mathbb{F}_{q}\left(\left(X^{-1}\right)\right)$ telle que $\operatorname{deg}[g] \geq 1$. Soit donc $f=T+1 / g$, qui vérifie l'équation $f^{2}=$ $T^{2}-S=Q$. Comme $Q$ n'est pas un carré parfait dans $\mathbb{F}_{q}[X]$, alors d'après le théorème 2 , toute racine carrée $f$ de $Q$ vérifie

$$
\operatorname{Per}(f) \leq\left(q^{\operatorname{deg} T}-1\right)^{2} q^{2 \operatorname{deg} T}<q^{2 \operatorname{deg} Q}
$$

et

$$
T(f) \leq \operatorname{deg} T=\frac{1}{2} \operatorname{deg} Q .
$$

Corollaire 3. Soient $q \geq 3$ et $A, B, C \in \mathbb{F}_{q}[X]$. Soient $\Delta=B^{2}-4 A C$ et $(\mathrm{E})$ l'équation algébrique $A Y^{2}+B Y+C=0$. Alors $(\mathrm{E})$ admet au moins une solution dans $\mathbb{F}_{q}\left(\left(X^{-1}\right)\right)$ si et seulement si $\operatorname{deg} \Delta$ est pair et $\sigma(\Delta)$ est un carré parfait.

Preuve. Il est clair que si $\operatorname{deg} \Delta$ est pair et $\sigma(\Delta)$ est un carré parfait, alors d'après le théorème $4, \Delta$ admet une racine carrée $\delta \in \mathbb{F}_{q}\left(\left(X^{-1}\right)\right)$. 
On vérifie facilement que les séries formelles $(-B \pm \delta) /(2 A)$ sont les seules solutions de (E).

Réciproquement, si $f$ est une solution de (E), il est clair que l'on a $\Delta=$ $(2 A f+B)^{2}$, ce qui donne que deg $\Delta$ est pair et que $\sigma(\Delta)$ est un carré parfait.

Par la suite $\Delta$ désigne le discriminant de la série formelle quadratique solution de (E).

Un nombre réel $x$ est dit quadratique s'il est irrationnel et vérifie une équation du type $a x^{2}+b x+c=0$ avec $a, b, c$ des entiers relatifs premiers entre eux. On note par la suite $\Delta(x)=b^{2}-4 a c$ le discriminant de $x$. Lewittes [6] a montré les deux théorèmes suivants :

ThÉorÈme. Soit $x$ un nombre réel quadratique. Alors les trois propriétés suivantes sont équivalentes:

(i) $x$ est palindromique.

(ii) $\operatorname{Norme}(x)=-1$.

(iii) $\Delta(x)$ est la somme de deux carrés.

ThÉORÈme. Soient $A(n)$ l'ensemble des nombres quadratiques $x$ dont le discriminant $\Delta(x)$ égal à $n$ et $w(n)$ le nombre de facteur premier de $n$. Si $16 \mid n$ ou si $n$ admet un facteur premier $\equiv 3(\bmod 4)$, alors $A(n)=0$. Si tout facteur premier de $n \equiv 1(\bmod 4)$, alors $A(n)=A(4 n)=2^{w(n)-1}$ et $A(8 n)=2^{w(n)}$.

Cela donne dans le cas des séries formelles le théorème 5 :

Démonstration du théorème 5. Soit $f$ une série formelle palindromique. Alors elle est de type (I), donc elle vérifie une équation de type $A f^{2}+$ $B f+C=0$ avec $\operatorname{deg} B>\operatorname{deg} A, \operatorname{deg} C$. D'après le lemme 1, si $f=$ $\left[a_{1} ; \overline{a_{2}, \ldots, a_{n}, a_{1}}\right]$, alors $(f+B / A)^{-1}=\left[a_{n} ; \overline{a_{n-1}, \ldots, a_{1}, a_{n}}\right]$. Puisque $f$ est palindromique, on a $f=(f+B / A)^{-1}$. D'autre part si $A(n, q)$ est le nombre de séries formelles palindromiques dont le degré du discriminant est égal à $2 n$, alors $A(n, q)$ est majoré par $B(n, q)$, le nombre de représentations des polynômes de degré $2 n$ sous la forme de la somme de deux carrés dont le degré $\leq n$. Mireille Car [2], [3] a donné une formule asymptotique de $B(n, q)$, à savoir : si $p \equiv 1(\bmod 4)$, alors

$$
B(n, q)=O\left(\frac{q^{2 n}}{n^{\alpha} \sqrt{\log 2 n}}\right) \quad \text { avec } \alpha=1-\frac{1+\log \log 2}{\log 2},
$$

et si $p \equiv 3(\bmod 4)$, alors

$$
B(n, q) \sim a \frac{q^{2 n}}{\sqrt{n \pi}} \quad \text { avec } a=\prod_{P \in I}\left(1-q^{-2 \operatorname{deg} P}\right)^{-1 / 2}
$$

et $I$ est l'ensemble de polynômes unitaires et irréductibles dans $\mathbb{F}_{q}[X]$. 


\section{Bibliographie}

[1] L. E. Baum and M. M. Sweet, Continued fraction of algebraic power series in characteristic 2, Ann. of Math. 103 (1976), 593-610.

[2] M. Car, Polynômes de $\mathbb{F}_{q}[X]$ ayant un diviseur de degré donné, Acta Arith. 43 (1984), 131-154.

[3] - Normes dans $\mathbb{F}_{q}[X]$ de polynômes de $\mathbb{F}_{q^{h}}[X]$, C. R. Acad. Sci. Paris 288 (1979), 669-672; Correction, ibid., 1049.

[4] H. Cohen, Multiplication par un entier d'une fraction continue périodique, Acta Arith. 26 (1974-75), 129-148.

[5] J. H. E. Cohn, The length of the period of the simple continued fraction of $d^{1 / 2}$, Pacific J. Math. 71 (1977), 21-32.

[6] J. Lewittes, Quadratic irrationals and continued fractions, in: Number Theory (New York, 1991-1995), Springer, New York, 1996, 253-268.

[7] M. Mkaouar, Sur le développement en fractions continues des séries formelles quadratiques sur $\mathbb{F}_{2}(X)$, J. Number Theory 80 (2000), 169-173.

[8] A. Schinzel, On some problems of the arithmetical theory of continued fractions. I, Acta Arith. 6 (1961), 394-413; II, ibid. 7 (1962), 287-298; Corrigendum, ibid. 47 (1986), 295.

Département de Mathématiques

Faculté des Sciences de Sfax

Route Soukra km 3,5

3038 Sfax-Tunisie

E-mail: mohamed.mkaouar@fss.rnu.tn 